\title{
黄抱原毛平革菌菌球对多环芳烃的生物吸附和 生物降解作用
}

$丁^{\text {洁 }^{(1)} \text {, 陈宝梁 }}{ }^{(12 *}$, 朱利中 ${ }^{(1) 2}$

(1) 浙江大学环境科学系, 杭州 310058 ;

(2) 浙江省有机污染过程与控制重点实验室，杭州 310058

* 联系人, E-mail: blchen@zju.edu.cn

2012-03-30 收稿, 2012-05-21 接受

国家自然科学基金(41071210)、国家高技术研究发展计划(2012AA06A203)和浙江省自然科学基金杰出青年基金(R5100105)资助

摘要 以黄胞原毛平革菌(Phanerochaete chrysosporium, $\mathrm{Pc}$ ) 为代表, 研究了死体和活体 $\mathrm{Pc}$ 菌 球对水中菲、萠等多环芳烃( PAHs) 的生物吸附和生物降解作用及其相对贡献, 探讨了碳源、 氮源浓度和共存 $\mathrm{Cu}^{2+}$ 的影响, 考察了连续多次输入下菲的生物吸附和生物降解作用, 试图 为准确掌握微生物作用下 PAHs 的迁移转化行为及其调控作用提供理论指导. 结果表明, 死 体 Pc 菌球对水中菲、萠的去除机制为生物吸附, 分配系数分别为 4040 和 $17500 \mathrm{~L} / \mathrm{kg}$. 活体 Pc 菌球的去除机制包括生物吸附和生物降解, $3 \mathrm{~d}$ 时生物吸附对水中菲、萠的去除率分别为 $19.71 \%$ 和 $52.21 \%$; 随着作用时间延长(3 40 d), 生物降解对菲、萠的去除作用逐渐增大 (20.40\% 60.62\%和 $15.55 \%$ 49.21\%), 且菌体中 PAHs 残留量不断下降. 富碳限氮营养条件 下, 活体 Pc 菌球对 PAHs 的去除作用和降解效果最优, 在 $60 \mathrm{~d}$ 时分别为 $99.55 \%$ 和 92.77\%(菲), 99.47\%和 83.97\%(萠), 主要源于富碳增加 Pc 菌体生物量而增强生物吸附、限氮 则触发 Pc 对 PAHs 的生物降解作用。活体 Pc 菌球在富碳限氮强化条件下可连续多次去除和 降解新输入的 PAHs, 其中单次生物降解率随循环次数 (6 d/次)增加而增大, 循环 3 次后菲的 生物降解率高达 $90 \%$.

关键词

生物吸附

生物降解

黄孢原毛平革菌

多环芳烃

生物修复
持久性有机污染物(POPs)具有毒性、生物累积和 长距离迁移等特性 ${ }^{[13]}$. 环境中 POPs 种类多、来源 广、分布普遍 ${ }^{[1,4,5]}$, 可通过呼吸、饮水、食物链危及 人体健康 ${ }^{[6]}$. POPs 的界面行为、环境过程、健康效应、 污染控制与修复已成为目前国内外环境领域的研究 热点之一 ${ }^{[6-10]}$. 微生物修复是最具应用潜力的治理 POPs 污染环境的绿色技术之一 ${ }^{[10 ~ 15] . ~}$

微生物修复中微生物体起生物吸附 (吸附介质) 和生物降解(反应器)的双重作用 ${ }^{[15]}$. 生物吸附是指将 化学物质吸附到生物表面或进人其内部的物化过 程 ${ }^{[16,17]}$. 目前, 用细菌、真菌、藻类等生物材料来去 除重金属、染料、杀虫剂等污染物已有大量报道 ${ }^{[16 ~ 20]}$,
但是有关 POPs 的生物吸附研究相对较少. 由于微生 物活体受营养元素、 $\mathrm{pH}$ 等条件的影响, 故常选择微 生物死体作为吸附材料用于实际污染控制中 ${ }^{[19 \sim 21]}$. 丁洁等人 ${ }^{[22]}$ 研究了白腐真菌死体对菲和萠的生物吸 附-脱附作用及构-效关系, 认为其吸附机理为有机污 染物在白腐真菌体上的分配作用, 吸附-脱附呈可逆 过程. 此外, 有机污染土壤微生物修复中更多关注生 物降解作用, 由于难以区分土壤复杂体系中生物吸 附和土壤介质吸附, 故有关微生物体的生物吸附及 对生物降解的影响很少研究 ${ }^{[15]}$.

近年来, 有关生物吸附和生物降解对环境中 POPs 的迁移转化行为及污染控制的作用逐渐引起关

英文版见: Ding J, Chen B L, Zhu L Z. Biosorption and biodegradation of polycyclic aromatic hydrocarbons by Phanerochaete chrysosporium in aqueous solution. Chin Sci Bull, 2012, 57, doi: 10.1007/s11434-012-5411-9 
注. Stringfellow 和 Alvarez-Cohen ${ }^{[23]}$ 研究认为生物吸 附能在短期内迅速去除水中多环芳烃(PAHs), 在一 定程度上降低了吸附态 PAHs 的生物降解作用. Chen 等人 ${ }^{[15]}$ 定量描述了活体白腐真菌对 PAHs 的生物吸 附、生物降解及相对贡献, 结合培养前后白腐真菌的 生物量变化, 活体白腐真菌对 PAHs 的表观分配系数 与死体白腐真菌分配系数比值 $\left(K_{\mathrm{d}}{ }^{*} / K_{\mathrm{d}}\right)$, 判别不同营 养条件下生物降解发生情况; 生物吸附减缓生物降 解的发生, 但是限氮和共存 PAHs 可促进生物降解. Yesilada 等人 ${ }^{[24]}$ 研究发现真菌菌球对染料的多次脱 色过程中, 死体菌球的脱色效果逐渐下降甚至为零, 而活体菌球的效果优于死体菌球, 生物吸附和生物 降解的共同作用可达到更好地污染削减效果. 有关 微生物体对有机污染物的生物吸附和生物降解的调 控因子及强化措施需深人研究, 有关微生物修复长 期运行中 POPs 的连续生物吸附和生物降解的研究几 乎为空白.

黄狍原毛平革菌 (Phanerochaete chrysosporium, Pc) 是一类典型的白腐真菌, 具有降解类木质素结 构的杂环类化合物 (如 PAHs)的能力 ${ }^{[25,26]}$. 白腐真菌 对底物要求低 ${ }^{[27,28]}$, 生物量大且对修复环境的适应 性强, 在 POPs 污染环境微生物修复中受到广泛关

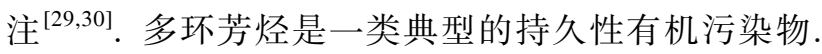
为此, 本文选用菲和芘为 PAHs 代表, Pc 为微生物材 料, 研究了死体和活体 Pc 菌球对水中 PAHs 的生物 吸附和生物降解作用及其相对贡献，探讨了碳源、氮 源浓度和共存 $\mathrm{Cu}^{2+}$ 的调控作用, 考察了连续多次输 人下菲的生物吸附和生物降解作用, 试图为准确掌 握微生物作用下 PAHs 的迁移转化行为及微生物修 复作用提供理论指导.

\section{1 实验材料与方法}

(i) 实验材料. 菲 $\left(\mathrm{C}_{14} \mathrm{H}_{10},>97 \%\right)$ 和萠 $\left(\mathrm{C}_{16} \mathrm{H}_{10}\right.$, $>98 \%$ )购自 Sigma-Aldrich 公司, 其相对分子量分别 为 178.2 和 202.3 , 水中溶解度为 1.18 和 $0.13 \mathrm{mg} / \mathrm{L}$ $\left(25^{\circ} \mathrm{C}\right)$, 正辛醇-水分配系数 $\left(K_{\mathrm{ow}}\right)$ 为 $2.8 \times 10^{4}$ 和 $8.0 \times 10^{4}$. 选用的 Pc 购自中国微生物菌种保藏中心, CGMCC 编 号为 5.0776. 菌种采用马丁氏固体培养基培养, 其基 本组成为 $0.5 \mathrm{~g} / \mathrm{L} \mathrm{MgSO}{ }_{4} \cdot 7 \mathrm{H}_{2} \mathrm{O}, 1 \mathrm{~g} / \mathrm{L} \mathrm{KH}_{2} \mathrm{PO}_{4}, 10 \mathrm{~g} / \mathrm{L}$ 葡萄糖, $5 \mathrm{~g} / \mathrm{L}$ 蛋白炼, $16 \mathrm{~g} / \mathrm{L}$ 琼脂. 菌种在 $35 \pm 0.5^{\circ} \mathrm{C}$ 下平板培养 $5 \mathrm{~d}$ 后, 用灭菌水冲洗平板表面获得孢子 悬液; 以 $10^{5} \mathrm{ceus} / \mathrm{mL}$ 的狍子悬液接种马丁氏液态培
养基, 于 $35 \pm 0.5^{\circ} \mathrm{C}, 120 \mathrm{r} / \mathrm{min}$ 条件下培养 $7 \mathrm{~d}$, 形成大 小均一, 直径约 $0.6 \mathrm{~cm}$ 的 Pc 菌球.

(ii) Pc 菌球对水中菲和萠的生物吸附与生物降 解实验. 实验分死体菌球组和活体菌球组, 活体菌 球选用同一批次培养的直径约 $0.6 \mathrm{~cm} \mathrm{Pc}$ 菌球; 而死 体菌球为经 $121^{\circ} \mathrm{C}$ 下高压蒸气灭菌 $20 \mathrm{~min}$ 后得到的 灭活菌球. 具体实验步骤如下: 在 $40 \mathrm{~mL}$ 样品瓶中, 先后加人 1 颗死体或活体 Pc 菌球, $15 \mathrm{~mL}$ 含 $1.0 \mathrm{mg} / \mathrm{L}$ 菲和 $0.1 \mathrm{mg} / \mathrm{L}$ 萠的混合溶液, 每组设置 3 个平行. 加 盖内垫锡箔纸和聚四氟乙烯垫片的盖子, 于 $35 \pm$ $0.5^{\circ} \mathrm{C}, 120 \mathrm{r} / \mathrm{min}$ 和避光条件下振荡, 每天通风 $2 \mathrm{~min}$ 以保证活体微生物生长所需的氧气, 分别于培养 3,7 , $14,40 \mathrm{~d}$ 时, 取出 $40 \mathrm{~mL}$ 样品瓶, $3000 \mathrm{r} / \mathrm{min}$ 离心 10 $\min$, 测定溶液中和菌球上菲和萠的含量. 取上层溶 液与乙腈混合 $(1: 1$, 体积比), 过 $0.22 \mu \mathrm{m}$ 滤膜后, 用 带苂光检测器(FLD)的高效液相色谱(HPLC)分析溶 液中菲和萠. 完全移去上层清液, 加人 $10 \mathrm{~mL}$ 丙酮正己烷混合液(1:1, 体积比), 超声 $30 \mathrm{~min}$ 后提取菌 球上的菲和萠; 重复 3 次, 合并收集萃取液, 加人 30 $\mu \mathrm{L}$ 二甲基亚砜, 于 $40^{\circ} \mathrm{C}$ 下旋转蒸发至干, 加人 $5 \mathrm{~mL}$ 正已烷定容. 取 $1 \mathrm{~mL}$ 溶液过 $2.5 \mathrm{~g}$ 的 60 100 目硅胶 柱, 用 $15 \mathrm{~mL}$ 二氯甲烷正己烷混合液 $(1: 1$, 体积比) 淋洗，收集淋洗液; 加人二甲基亚砜后再次旋转蒸发 至干, 然后加人 $4 \mathrm{~mL}$ 乙腈定容, 过 $0.22 \mu \mathrm{m}$ 滤膜, 用 HPLC-FLD 测定. HPLC 的测定条件: $90 \%$ 乙腈-10\%水 (体积比)为流动相, 流速 $1.0 \mathrm{~mL} / \mathrm{min}$, 柱温 $30^{\circ} \mathrm{C}$, 采 用苂光检测器, 菲和萠的激发波长为 244 和 $237 \mathrm{~nm}$, 发射波长为 360 和 $385 \mathrm{~nm}$. 菌球萃取 PAHs 后, $40 \mathrm{~mL}$ 样品瓶于 $65^{\circ} \mathrm{C}$ 下烘干 $8 \mathrm{~h}$, 通过实验前后瓶子的质量 差计算菌球干重. 设置不加菌球的空白对照组, 以测 定挥发可能产生的损失. 所有操作均在无菌条件下 进行. 用吸附率 $(S)$ 、降解率 $(D)$ 和去除率 $(R)$ 来表示生 物吸附、生物降解以及两者共同作用对水中 PAHs 的 去除效果，计算公式如下：

$$
\begin{gathered}
R=\left(C_{0}-C_{\text {soln }}\right) / C_{0} \times 100 \%, \\
S=m_{\mathrm{p}} /\left(C_{0} V\right) \times 100 \%, \\
D=(R-S) \times 100 \%,
\end{gathered}
$$

式中 $C_{0}(\mu \mathrm{g} / \mathrm{mL})$ 表示溶液中 PAHs 的初始浓度, $C_{\text {soln }}(\mu \mathrm{g} / \mathrm{mL})$ 为不同培养期溶液中 PAHs 浓度, $m_{\mathrm{p}}(\mu \mathrm{g})$ 为菌球吸附 PAH 的质量, $V(\mathrm{~mL})$ 表示溶液体积.

（iii）碳源、氮源及接种孢子浓度对 PAHs 的生 物吸附和生物降解的影响. 碳源和氮源是 Pc 生长所 
必需的营养元素, 同时也是酶分泌的重要调控因 子 ${ }^{[31,32]}$, 进而影响 PAHs 的生物吸附和生物降解. 葡 萄糖是最常见的碳源, 而 Pc 对于氮源类型的敏感度 很低 ${ }^{[33]}$, 因此选择常见的硝酸钠为氮源. 实验使用 优化的马丁氏培养液, 基本组成为: $0.5 \mathrm{~g} / \mathrm{L} \mathrm{MgSO}_{4}$. $7 \mathrm{H}_{2} \mathrm{O}, 1 \mathrm{~g} / \mathrm{L} \mathrm{KK_{2 }} \mathrm{PO}_{4}$, 葡萄糖为碳源, $\mathrm{NaNO}_{3}$ 为氮源, 培养液 $\mathrm{pH}$ 控制在 $4.5 \pm 0.1 . \mathrm{NaNO}_{3}$ 浓度为 2.4 $\mathrm{mmol} / \mathrm{L}$ (限氮组), $24 \mathrm{mmol} / \mathrm{L}$ (富氮组); 葡萄糖浓度为 $5.6 \mathrm{mmol} / \mathrm{L}$ (限碳组), $56 \mathrm{mmol} / \mathrm{L}$ (富碳组 $)^{[33]}$; 接种孢 子浓度分别为 $10^{5}$ 和 $10^{6}$ ceus $/ \mathrm{mL}$. 通过 $\mathrm{L}_{8}\left(2^{7}\right)$ 的正交 试验(表 1), 来探讨碳浓度、氮浓度、接种狍子的浓 度及其两两交互作用对生物吸附和生物降解的影响. 根据表 1 的设置, 在 $40 \mathrm{~mL}$ 样品瓶中加人 $1.0 \mathrm{~mL}$ 孢 子悬液, 再加人 $20 \mathrm{~mL}$ 含 $1.0 \mathrm{mg} / \mathrm{L}$ 菲和 $0.1 \mathrm{mg} / \mathrm{L}$ 芘 的培养液. 同时设置不接种狍子, 并且添加 $\mathrm{NaN}_{3}$ 的 抑菌组作为空白对照, 培养条件同上. 分别于 10 和 $60 \mathrm{~d}$ 后测定溶液和菌体中菲、萠的浓度，同时测定培 养后微生物菌体干重.

(iv) 连续培养多次添加下 $\mathrm{Pc}$ 对菲的生物吸附和 生物降解实验。研究了不同浓度碳源和氮源、 $\mathrm{Cu}^{2+}$ 离子共存下, 连续培养多次添加下菲的生物吸附和 生物降解过程. 实验在 $40 \mathrm{~mL}$ 样品瓶中进行, 共设置 8 个组( $\mathrm{A} \sim \mathrm{H}$ 组), 即 $\mathrm{A}$ 组: 死体菌球+抑菌剂 $\mathrm{NaN}_{3}$ ( $200 \mathrm{mg} / \mathrm{L})$; B 组: 死体菌球 $+0.25 \mathrm{mmol} / \mathrm{L} \mathrm{CuCl}{ }_{2}$; 组: 活体菌球; $\mathrm{D}$ 组：活体菌球 $+56 \mathrm{mmol} / \mathrm{L}$ 葡萄糖; $\mathrm{E}$ 组: 活体菌球 $+2.4 \mathrm{mmol} / \mathrm{L} \mathrm{NaNO}_{3} ; \mathrm{F}$ 组: 活体菌球 +56 $\mathrm{mmol} / \mathrm{L}$ 葡萄糖 $+2.4 \mathrm{mmol} / \mathrm{L} \mathrm{NaNO}_{3} ; \mathrm{G}$ 组: 活体菌球 $+0.25 \mathrm{mmol} / \mathrm{L} \mathrm{CuCl}{ }_{2} ; \mathrm{H}$ 组: 活体菌球 $+56 \mathrm{mmol} / \mathrm{L}$ 葡 萄糖+ $0.25 \mathrm{mmol} / \mathrm{L} \mathrm{CuCl}_{2}$. 每组设置 15 个平行. 多次

表 1 碳源、氮源及孢子浓度对 PAHs 生物吸附和生物降解 影响的正交试验设计

\begin{tabular}{ccccc}
\hline \multirow{2}{*}{$\begin{array}{c}\text { 组 } \\
\text { 别 }\end{array}$} & $\begin{array}{c}\text { 影响因素 } \\
(\mathrm{mmol} / \mathrm{L})\end{array}$ & $\begin{array}{c}\text { 氮源浓度萄糖碳源浓度 } \\
(\mathrm{mmol} / \mathrm{L})\end{array}$ & $\begin{array}{c}\text { 孢子浓度 } \\
(\mathrm{ceus} / \mathrm{mL})\end{array}$ & $\begin{array}{c}\text { 生物量 } \\
(\mathrm{mg})\end{array}$ \\
\hline 1 & 2.4 & 5.6 & $10^{5}$ & 3.54 \\
2 & 2.4 & 5.6 & $10^{6}$ & 3.44 \\
3 & 2.4 & 56 & $10^{5}$ & 15.98 \\
4 & 2.4 & 56 & $10^{6}$ & 16.23 \\
5 & 24 & 5.6 & $10^{5}$ & 4.63 \\
6 & 24 & 5.6 & $10^{6}$ & 5.14 \\
7 & 24 & 56 & $10^{5}$ & 17.48 \\
8 & 24 & 56 & $10^{6}$ & 17.92 \\
\hline
\end{tabular}

a) 培养 $60 \mathrm{~d}$ 后 Pc 菌体的干重
添加、连续培养实验过程如下: 添加 $15 \mathrm{~mL} 1.0 \mathrm{mg} / \mathrm{L}$ 的菲溶液, 加盖内垫锡箔纸(对含 $\mathrm{CuCl}_{2}$ 组则不垫锡 箔纸, 以防发生置换反应, 该操作对实验结果不影响) 和聚四氟乙烯垫片的盖子, 于 $35 \pm 0.5^{\circ} \mathrm{C}, 120 \mathrm{r} / \mathrm{min}$, 避光条件下振荡, 每天通风 $2 \mathrm{~min}$; 每培养 $6 \mathrm{~d}$ 后, 取 出 $\mathrm{A} \sim \mathrm{H}$ 各组 3 个平行样品, 测定溶液和菌球中菲的 浓度、菌球干重, 计算菲的生物去除、生物吸附和生 物降解; A H 各组中其余平行样品, 完全倒干样品瓶 中的溶液后, 重新加人 $15 \mathrm{~mL} 1.0 \mathrm{mg} / \mathrm{L}$ 的菲溶液及 相应强化因子, 再连续培养 $6 \mathrm{~d}$; 如此循环 5 次, 分别 在多次添加、连续培养 $6,12,18,24,30 \mathrm{~d}$ 后, 测定溶 液和菌球中菲的浓度、菌球干重, 计算第 1 5 次添加 后菲的单次生物去除百分率 $(\%)$ 、单次的生物降解百 分率 $(\%)$ 和单次的生物吸附累积量 $(\mu \mathrm{g})$. 同时设置一 组不添加任何强化因子的活体组 (组成同 $\mathrm{C}$ 组), 单次 添加菲而不做连续运行实验作为对照实验 $(\mathrm{CK})$, 测 定非连续运行条件下 Pc 对菲的生物吸附和生物降解, 每个时间点设置 3 个平行, 每隔 $6 \mathrm{~d}$ 与 $\mathrm{A} \sim \mathrm{H}$ 组同时测 定菲的浓度. 在连续运行过程中, $\mathrm{Pc}$ 对溶液中菲的单 次去除率计算如下:

$$
R_{x}=\left(C_{0 x}-C_{x}\right) / C_{0 x} \times 100 \%,
$$

式中, $R_{x}$ 表示连续运行第 $x$ 次时的单次去除率, $C_{0 x}(\mu \mathrm{g} / \mathrm{mL})$ 表示连续运行第 $x$ 次时所加菲的溶液的初 始浓度, $C_{x}(\mu \mathrm{g} / \mathrm{mL})$ 表示连续运行第 $x$ 次时 $6 \mathrm{~d}$ 后测得 的溶液中菲的浓度. 单次吸附量(或残留量)由吸附总 量与前一次残留量之差表示, 单次残留量为正值表 示溶液中的菲通过吸附去除，为负值表示有部分前 一次残留的菲脱附或降解. 单次降解量通过质量平 衡计算, 单次降解率表示单次降解量占此次所加菲 总量的百分比值.

\section{2 结果与讨论}

\section{$2.1 P c$ 菌球对菲、萠的生物吸附和生物降解作用}

死体和活体 Pc 菌球对水溶液中菲和芘的去除作 用见图 1. 死体 Pc 菌球对菲和萠的吸附作用在 $3 \mathrm{~d}$ 内 已达到平衡, 去除率分别为 $40.23 \% \pm 0.11 \%$ 和 $74.71 \% \pm 0.60 \%$. 已有研究表明, 微生物死体对非极 性有机污染物的吸附机制为分配作用 ${ }^{[15,22]}$. 因此, 计 算得到的死体 $\mathrm{Pc}$ 对菲和萠的分配系数 $\left(K_{\mathrm{p}}\right)$ 分别为 $4040 \pm 170$ 和 $17500 \pm 1000 \mathrm{~L} / \mathrm{kg}$ ，与取自废弃木材上 的白腐真菌培养成的菌球死体的吸附性能相近 ${ }^{[22]}$, 


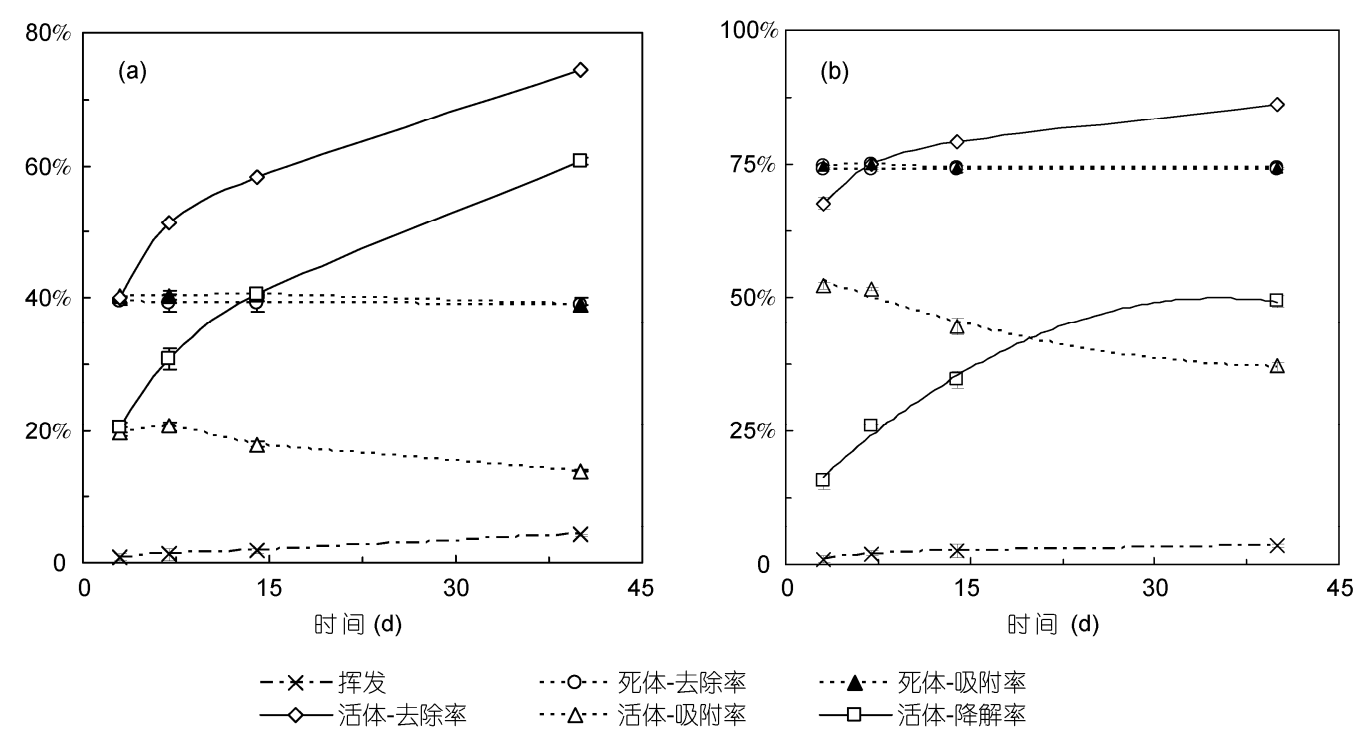

图 1 无营养条件下 Pc 菌体对菲(a)和萠(b)的生物吸附和生物降解作用

即 $K_{\mathrm{p}}=3721 \mathrm{~L} / \mathrm{kg}$ (菲)和 $15980 \mathrm{~L} / \mathrm{kg}$ (萠). 萠在微生物 体上的吸附能力强于菲, 与它们的 $K_{\mathrm{ow}}$ 大小一致. 死 体菌球对溶液中 PAHs 的去除百分率与从菌体上萃 取测得的吸附百分率一致，并且在实验时间范围内 $(3 \sim 40 \mathrm{~d})$ 去除率都维持在稳定状态, 说明生物吸附是 死体菌球去除溶液中菲和萠的唯一途径. 在 3 40 d 内, 挥发引起的 PAHs 的损失很小, 分别为 $4.17 \% \pm$ $0.11 \%$ (菲)和 $3.55 \% \pm 0.31 \%$ (芘).

活体 Pc 菌球在培养 $3 \mathrm{~d}$ 时对溶液中菲的去除率 为 $40.11 \%$, 与死体菌球的去除率(40.23\%)基本相同; 而对萠, $3 \mathrm{~d}$ 时活体菌球的去除率为 $67.76 \%$, 低于死 体的去除率 $(74.71 \%)$. 随着培养时间的延长, 活体 Pc 菌球对溶液中菲和萠的去除率逐渐增大, 到 $40 \mathrm{~d}$ 时 去除率分别达 $74.44 \%$ 和 $86.24 \%$, 远高于死体菌球的 去除率. 活体 Pc 菌球对菲和萠的去除作用包括生物 吸附和生物降解两种途径. $3 \mathrm{~d}$ 时, 活体 Pc 菌球对菲 和萠的生物吸附去除率为 $19.71 \%$ 和 $52.21 \%$, 远低于 死体菌球的生物吸附去除率, 这主要归因于高压灭 活改变 Pc 细胞的通透性所致 ${ }^{[34]}$; 而随着培养时间延 长, 活体菌球的生物吸附去除率逐渐降低, 到 $40 \mathrm{~d}$ 时, 分别为 $13.82 \%$ 和 $37.03 \%$. 与生物吸附作用相反, 生 物降解作用随培养时间延长而逐渐增大, 3 40 d 内, 菲和萠的生物降解分别为 $20.40 \% \pm 0.73 \%$ 60.62\% $0.56 \%$ 和 $15.55 \% \pm 1.57 \% \sim 49.21 \% \pm 1.09 \%$, 且菲的生 物降解速率比萠快. 比较生物吸附、生物降解和生物 去除随培养时间的变化规律可得, 活体 Pc 菌球对菲
和萠去除作用随时间而增大的贡献主要来自生物降 解作用. 同时, Pc 活体菌球中菲和萠的吸附量逐渐降 低(下降 $5.89 \%$ 和 $15.18 \%$ ), 说明溶液和菌体内的菲和 萠均可发生生物降解作用. 由于微生物体对 PAHs 的 吸附由分配作用主导、且吸附-脱附过程是可逆 的 ${ }^{[15,22]}$, 随着体系中 PAHs 总量的减少, 吸附在菌体 上的 PAHs 可能脱附出来而发生降解, 也可在菌体上 直接发生降解作用.

多环芳烃的生物吸附是环境中非常普遍的现象. 微生物修复作为治理 PAHs 污染最有前途的修复技 术之一 ${ }^{[12,13]}$, 微生物本身对 PAHs 的生物吸附没有引 起足够的重视. 而实际上生物吸附对表观生物去除 有较大贡献, 如 $3 \mathrm{~d}$ 时活体 Pc 菌球通过生物吸附对溶 液中菲和萠的去除率分别达到 $19.71 \%$ 和 $52.21 \%$, 使 PAHs 迅速由溶解态转为吸附态 ${ }^{[15]}$. 由于实验中培养 液不添加任何营养元素和微量元素, Pc 菌球的生物 量增长几乎可以忽略, 培养 $40 \mathrm{~d}$ 后活体菌球和死体 菌球的质量相当, 分别为 $2.22 \pm 0.05$ 和 $2.25 \pm 0.12 \mathrm{mg}$. 但是在生物降解参与下, 活体菌球组的表观去除率 高于死体组, 且有 $60.62 \%$ 菲和 $49.21 \%$ 萠通过生物降 解途径从体系中去除.

\section{2 不同营养条件对 PAHs 在 Pc 菌体上的生物吸 附和生物降解作用的影响}

碳源和氮源在不同浓度条件下 (见表 1)，活体 Pc 对菲和萠的生物吸附和生物降解情况如图 2 所示. 溶 
液中菲和萠主要去除途径包括生物吸附和生物降解, 对于富碳 (56 mmol/L 葡萄糖)、富氮 (24 mmol/L $\mathrm{NaNO}_{3}$ )、1 $10^{6}$ 孢子浓度接种的第 8 组, $10 \mathrm{~d}$ 内 $54.25 \%$ 的菲和 $59.30 \%$ 的萠通过生物吸附去除，而有 $32.16 \%$ 的菲和 $30.85 \%$ 的萠通过生物降解去除; 而 $60 \mathrm{~d}$ 时, 大部分的菲和芘都通过生物降解而去除, 去除率分 别达到 $84.77 \%$ 和 $77.96 \%$, 残留的菲和萠主要以菌体 吸附态形式存在, 溶解态则非常少. 碳源是影响菲、 萠生物去除的重要因素, 从图 2 中可以看出, 富碳的 第 3, 4, 7, 8 组的去除率明显优于其余缺碳各组, 这主 要与富碳时 $\mathrm{Pc}$ 的生物量较大直接有关 (见表 1). 活体 $\mathrm{Pc}$ 生物量增大, 使产生生物吸附和生物降解的 Pc 的 量增多. 通过方差分析发现, 氮源浓度和碳源浓度对 生物量影响的 $F$ 值分别为 119.89 和 8672.57, 说明碳 源、氮源浓度对 Pc 生物量都有显著性的影响, 且碳 源对 Pc 生物量的影响远远大于氮源的影响.

虽然生物吸附和生物降解都可作为水溶液中
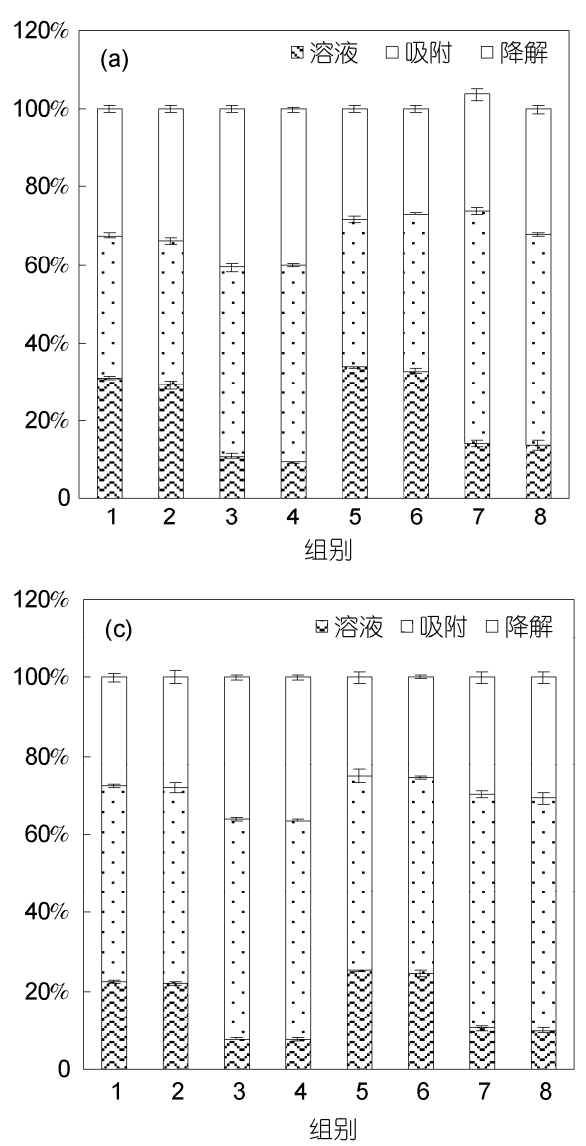

PAHs 生物消去的有效途径, 但是生物吸附其实是 PAHs 在微生物体固相上的积累储存过程, 生物降解 才是真正消去, 因此, 预测环境中 POPs 的迁移转化 行为和优化生物修复条件时, 需要区分生物吸附和 生物降解及相对贡献. 表 2 列出了碳源、氮源和接种 孢子的浓度对菲的生物去除、生物吸附和生物降解 的正交分析数据, 结果表明, 碳源、氮源对生物吸附 和生物降解的影响较为复杂, 不同培养时间其影响 效果也不同. 碳源浓度对菲的去除率的影响非常显 著, 这与碳源浓度对 Pc 生物量起关键调控作用有关. 氮源浓度的影响相对复杂些, 从长期培养 $(60 \mathrm{~d})$ 看, 氮源浓度的影响并不显著, 而在短期 $(10 \mathrm{~d})$ 内氮源浓 度的影响却非常显著, 这是由于氮源是控制生物量生 长的重要因素, 更重要的是在短期中, 氮饥饿是 $\mathrm{Pc}$ 降 解 PAHs 的触发因子 ${ }^{[33,35]}$. 第 4 组(富碳、限氮、 $10^{6}$ 孢 子浓度)和第 8 组(富碳、富氮、 $10^{6}$ 孢子浓度)在 $10 \mathrm{~d}$ 内对菲去除率分别为 $90.83 \%$ 和 $86.41 \%$, 培养 $60 \mathrm{~d}$ 时则
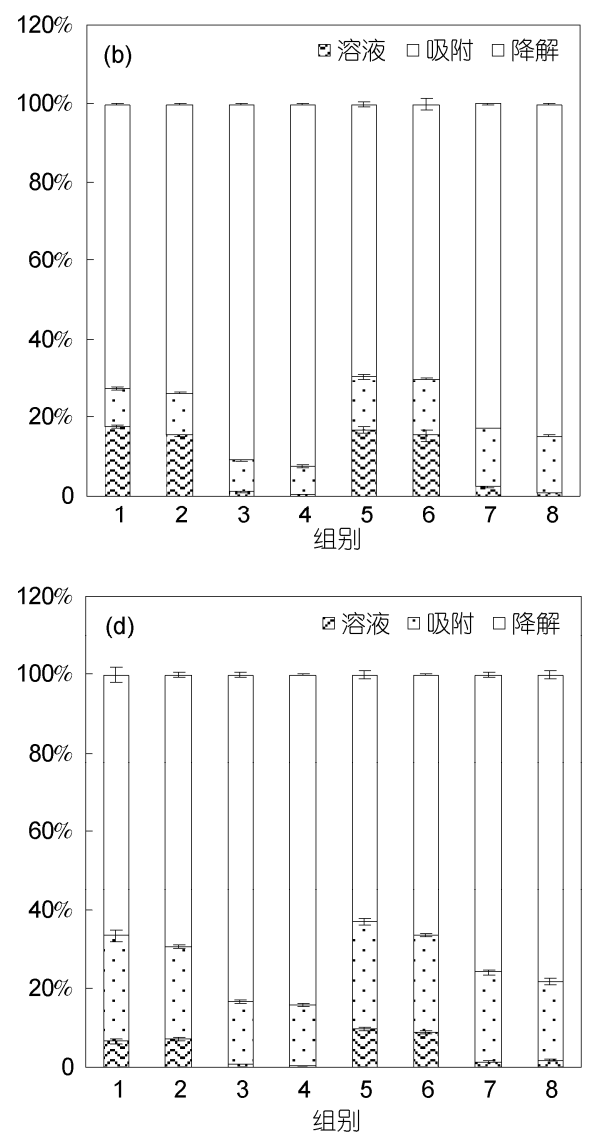

图 2 不同营养条件下黄狍原毛平革菌活体对溶液中菲和芘的生物去除、生物吸附和生物降解作用 (a) 菲-培养 $10 \mathrm{~d}$, (b) 菲-培养 $60 \mathrm{~d}$, (c) 芘-培养 $10 \mathrm{~d}$, (d) 芘-培养 $60 \mathrm{~d}$. 误差棒位于柱状图上方, 1 8 组的营养条件见表 1 
分别为 $99.55 \%$ 和 $99.12 \%$, 在受试实验中去除率最大.

由表 2 可见，碳源和氮源浓度及其交互作用都对 $\mathrm{Pc}$ 的生物降解有显著性影响, 其中短期 $(10 \mathrm{~d})$ 内氮源 浓度影响的 $F$ 值(96.48)大于碳源浓度影响的 $F$ 值 (55.03), 支持限制氮浓度可触发 Pc 对菲的降解作用; 而长期(60 d)时, 氮源影响的 $F$ 值(173.3)又小于碳源 影响的 $F$ 值(1580), 但氮源的影响仍是一个显著性因 素 $\left(F_{\mathrm{N}}>F_{0.01}\right)$. 结合生物降解的数值, $60 \mathrm{~d}$ 时第 4 组和 第 8 组的活体 Pc 菌体对菲的降解率分别为 $92.77 \%$ 和 $82.05 \%$, 虽然此时两组的表观去除率相当, 但是限 氮组中有更多的菲发生生物降解而真正意义上被去 除, 而全氮组则有更多的菲只是转移到了生物体固 相中(生物吸附), 因此, 第 4 组(富碳、限氮)的去除率 和降解率最优. 对污染物萠而言, $60 \mathrm{~d}$ 内的去除率和 降解率则为 $99.47 \%$ 和 $83.97 \%$. 在短期(10 d)内各因素 对生物吸附作用的影响主要受碳源浓度调控, 这是 由于碳源浓度是调控生物量的决定性因素; 而长期 (60 d)内, 碳源浓度、氮源浓度及其交互作用都对生 物吸附有影响, 这是由于菲在 Pc 体内的残留量受生 物降解的影响. 在限氮、富碳、 $10^{6}$ 孢子浓度接种的 情况下(第 4 组), Pc 菌体对 PAHs 的去除作用和降解 作用最优.

表 2 碳源、氮源、接种孢子浓度对菲的生物去除、生物 吸附和生物降解的正交分析表 ${ }^{\text {a) }}$

\begin{tabular}{|c|c|c|c|c|c|}
\hline & \multirow{2}{*}{ 方差来源 } & \multicolumn{2}{|c|}{$10 \mathrm{~d}$} & \multicolumn{2}{|c|}{$60 \mathrm{~d}$} \\
\hline & & $F$ 值 & 显著性 & $F$ 值 & 显著性 \\
\hline \multirow{4}{*}{ 生物去除 } & $\mathrm{A}(\mathrm{N}$ 的浓度 $)$ & 174.9 & $* * *$ & 0.87 & \\
\hline & $\mathrm{B}(\mathrm{C}$ 的浓度 $)$ & 5144 & $* * *$ & 1969 & $* * *$ \\
\hline & $A^{*} B$ & 0.70 & & 4.53 & \\
\hline & $\mathrm{C}$ (孢子浓度) & 19.17 & $* *$ & 19.79 & $* *$ \\
\hline \multirow{4}{*}{ 生物吸附 } & $\mathrm{A}(\mathrm{N}$ 的浓度) & 10.38 & $* *$ & 155.9 & $* * *$ \\
\hline & $\mathrm{B}(\mathrm{C}$ 的浓度 $)$ & 155.4 & $* * *$ & 10.83 & $* *$ \\
\hline & $A * B$ & 2.07 & & 20.58 & $* *$ \\
\hline & $\mathrm{C}$ (孢子浓度) & 0.63 & & 0.49 & \\
\hline \multirow{4}{*}{ 生物降解 } & $\mathrm{A}(\mathrm{N}$ 的浓度 $)$ & 96.48 & $* * *$ & 173.3 & $* * *$ \\
\hline & $\mathrm{B}(\mathrm{C}$ 的浓度 $)$ & 55.03 & $* * *$ & 1580 & $* * *$ \\
\hline & $A * B$ & 6.29 & $*$ & 37.38 & $* * *$ \\
\hline & $\mathrm{C}$ (孢子浓度) & 0.16 & & 17.19 & $* *$ \\
\hline
\end{tabular}

a) 在所有方差分析表中, $\mathrm{A} * \mathrm{C}, \mathrm{A} * \mathrm{~B}$ 的离差平方和 $S$ 均小于误 差列, 说明 $\mathrm{A}$ 和 C, B 和 C 之间的交互作用可以忽略不计, 此两列 亦当作误差列. $F_{0.01}(1,3)=34.12, F_{0.05}(1,3)=10.13, F_{0.1}(1,3)=5.54$. * 表示 $F_{0.05}>F>F_{0.1}$, 有影响但不显著; **表示 $F_{0.01}>F>F_{0.05}$, 影响较 为显著; ***表示 $F>F_{0.01}$, 有显著性影响

\section{$2.3 P c$ 对菲的连续多次生物吸附和生物降解}

实验采用预先培养的菌球, 以迅速引人较大的 生物量, 增强 Pc 对环境的适应能力 ${ }^{[24]}$. 在 8 种条件 下 (A H 组), Pc 对菲的连续生物吸附和生物降解情况 见图 3. 死体 Pc 菌球 (A 组)对菲的单次去除率随运行 次数的增加而不断下降(图 3(b)), 第 1 次运行时对菲 的去除率为 $38.69 \% \pm 0.76 \%$, 连续运行 5 次后, 对菲 的去除率下降为 $0.79 \% \pm 0.36 \%$, 这主要是由于死体 Pc 菌球通过生物吸附去除了溶液中的菲。已有研究 表明, $\mathrm{Cu}^{2+}$ 能促进白腐真菌对 $\mathrm{PAHs}$ 的生物吸附作 用 ${ }^{[22]}$. 比较图 3(b)中的 A 和 B 组可知, 在连续吸附中 $\mathrm{Cu}^{2+}$ 的存在都促进死体 $\mathrm{Pc}$ 菌球(图 3(b), B 组)对菲的 生物吸附, 但是促进效果不大; 在第 1 次运行时 $\mathrm{A}$ 组 (不含 $\mathrm{Cu}^{2+}$ ) 的去除率为 $38.69 \%$, 而 $\mathrm{B}$ 组 $\left(\right.$ 含 $\mathrm{Cu}^{2+}$ ) 的去 除率为 $40.77 \%$, 在第 5 次时 $\mathrm{A}$ 组和 B 组的去除率分 别仅为 $0.79 \%$ 和 $2.26 \%$. 由此可见, 微生物死体吸附 作用受其吸附容量的限制无法适应连续运行, 可以 推测使用微生物死体处理 PAHs 污染废水时, 为满足 去除率的需求, 必将产生大量的含 PAHs 的生物污泥, 污泥排放后, 易造成 POPs 二次污染.

由图 3(b)可以看出, 无营养条件下活体 Pc 菌球 (C 组)对菲的单次去除率与 $\mathrm{A}, \mathrm{B}$ 组一样, 均随循环次 数增加而逐渐下降，在第 1 和第 5 次活体 $\mathrm{Pc}$ 菌球对 菲的去除率分别为 $62.35 \%$ 和 $24.06 \%$, 但仍高于死体 菌球(A 组)的作用 $(38.69 \%$ 和 $0.79 \%)$. 由图 3(a)可见, 无营养条件下活体 $\mathrm{Pc}$ 菌球 $(\mathrm{C}$ 组)的生物量与死体 $\mathrm{Pc}$ 菌球 (A 组和 B 组)的生物量相当, 可以说明除生物吸 附外生物降解参与了 $\mathrm{C}$ 组中菲的生物消去作用, 增 大了活体菌球对菲的去除率. 图 3(c)中虚线表示无营 养条件下活体 Pc 对菲的初始生物降解能力, 发现以 后各次的生物降解率都大于第一次的降解率，说明 在连续运行的过程中 $\mathrm{C}$ 组中 $\mathrm{Pc}$ 对菲的降解能力有一 定程度的提高. 因此, $\mathrm{C}$ 组对菲的表观去除率随循环 次数增加而下降的原因主要是由于生物吸附的贡献 下降造成。

考察了 $\mathrm{D} \sim \mathrm{H}$ 组碳源、氮源以及 $\mathrm{Cu}^{2+}$ 对连续运行 中活体 Pc 菌球的生物吸附和生物降解作用的影响, 其中 $\mathrm{F}$ 组 (富碳、限氮强化)在连续批次运行过程中单 次去除率最高, 在连续 5 次运行中都保持在 $81 \%$ 以上, 第 5 次运行时仍高达 $89.74 \%$, 说明碳、氮的强化作用 是提高连续运行中菲去除率的重要措施. 一方面, 这 是由于碳源是影响 Pc 生物量的控制因子, 如图 3(a) 

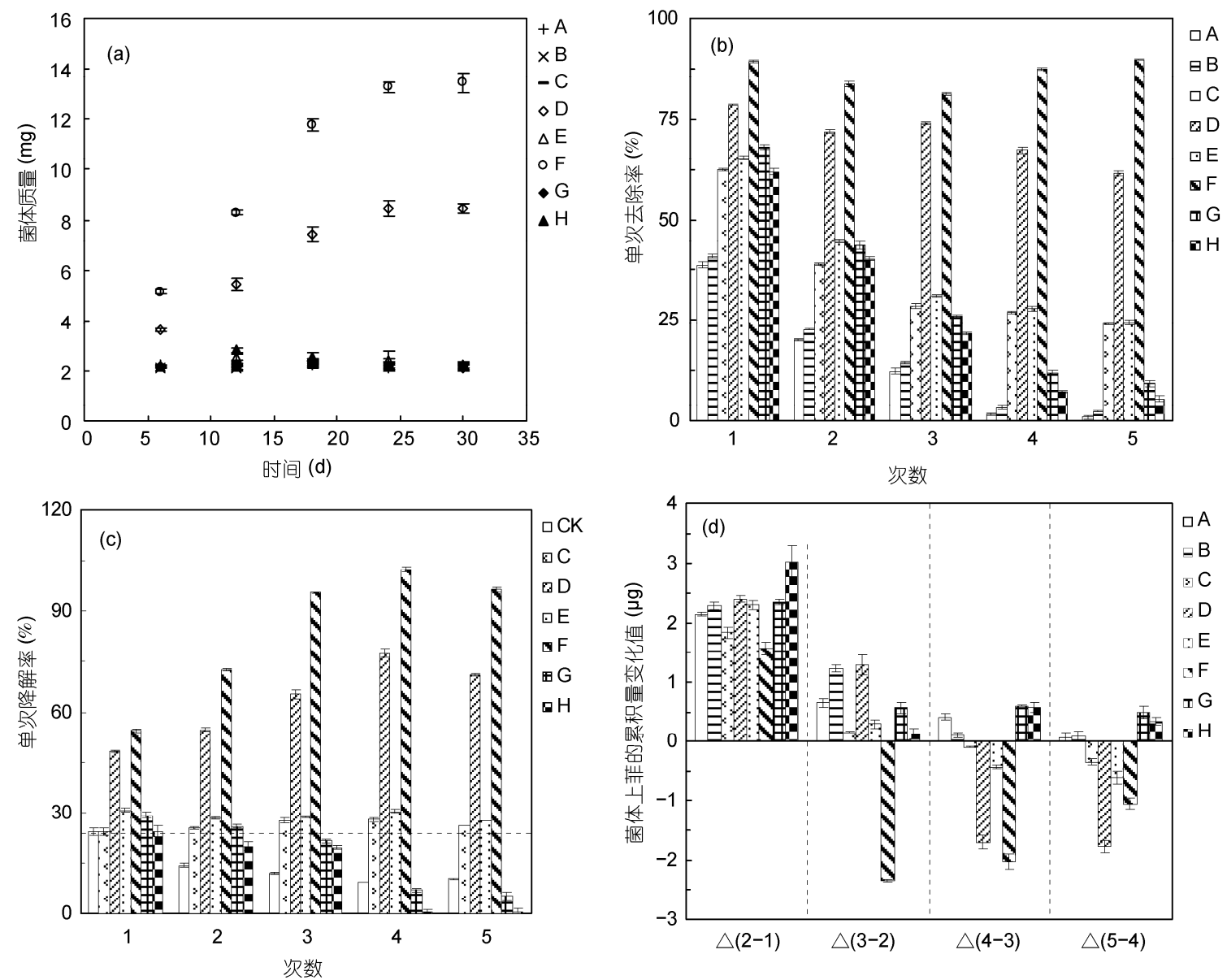

图 3 Pc 对菲的连续生物吸附和生物降解

(a) Pc 的生物量, (b) 菲的单次去除率, (c) 菲的单次生物降解率, (d) 与前一次运行后 Pc 菌体中菲的吸附量变化值(累积量). 1 5 表示 运行次数, $\mathrm{A} \sim \mathrm{H}$ 表示培养条件, 对照实验 $\mathrm{CK}$ 表示未进行批次运作时单次投加菲溶液的降解量

所示, 经碳源强化的 $\mathrm{D}$ 组和 $\mathrm{F}$ 组生物量增加明显, 而 同时用富碳、限氮强化的 $\mathrm{F}$ 组生物量最大, 在 $60 \mathrm{~d}$ 时达到 $13.44 \pm 0.38 \mathrm{mg}$, 明显大于菌球的初始质量 (约 $2.22 \mathrm{mg}$ ); 另一方面, 碳源、氮源的强化作用对生 物降解的促进作用明显(图 3(c)), 从第 1 次到第 3 次 循环, $\mathrm{F}$ 组的单次降解率逐渐增大, 从第 3 次开始保 持在 $90 \%$ 以上, 即每次运行过程中新输人的 $90 \%$ 的 菲都通过生物降解去除, 实现了稳定的生物消去作 用. 与死体菌球不同, 活体组得以连续运行的重要原 因是生物降解不仅降低了溶液中菲的浓度, 同时也 降低了菌球中残留的菲的浓度. 从图 3(d) 可以看出, 自第 3 次开始, $\mathrm{F}$ 组(富碳、限氮)中两次吸附量差量 即为负值, 即在连续操作过程中虽然仍有新的菲溶 液输人, 但是吸附在菌体内的菲的质量反而在不断 减小, 这保证了连续运行过程中的菲的低残留率. 在
第 5 次循环时, 包括未强化的 $\mathrm{C}$ 组也发生了残留在菌 体内的菲通过生物降解减少的现象, 这说明生物降 解在活体菌球组都发挥了重要作用, 使得活体菌球 组对菲的去除率普遍高于死体菌球. Keyser 等人 ${ }^{[33]}$ 研究表明, Pc 生长的生命周期可以分为生长期、繁殖 期和衰亡期 3 个阶段，对 PAHs 等污染物的次级代谢 过程发生在繁殖期. 不断引人的新鲜的 PAHs 溶液以 及强化组引人的碳、氮等营养元素均有助于维持 Pc 的次级代谢, 在 $30 \mathrm{~d}$ 实验周期中保持很高的降解.

$\mathrm{Cu}^{2+}$ 能促进 $\mathrm{Pc}$ 对菲的吸附, 同时 $\mathrm{Cu}^{2+}$ 对 $\mathrm{Pc}$ 降解 菲也有的一定的促进作用 ( $\mathrm{G}$ 组), $\mathrm{G}$ 组在第 1 次时对 菲的降解率达到 $29.29 \% \pm 0.96 \%$, 大于不含 $\mathrm{Cu}^{2+}$ 的 C 组 $(24.28 \% \pm 1.02 \%)$. 但是在连续运行过程中, 由于 $\mathrm{Cu}^{2+}$ 在 $\mathrm{Pc}$ 上的累积量增大, 微生物的生物量没有增 加, 表现出一定的毒害作用, 使得单次去除率逐渐下 


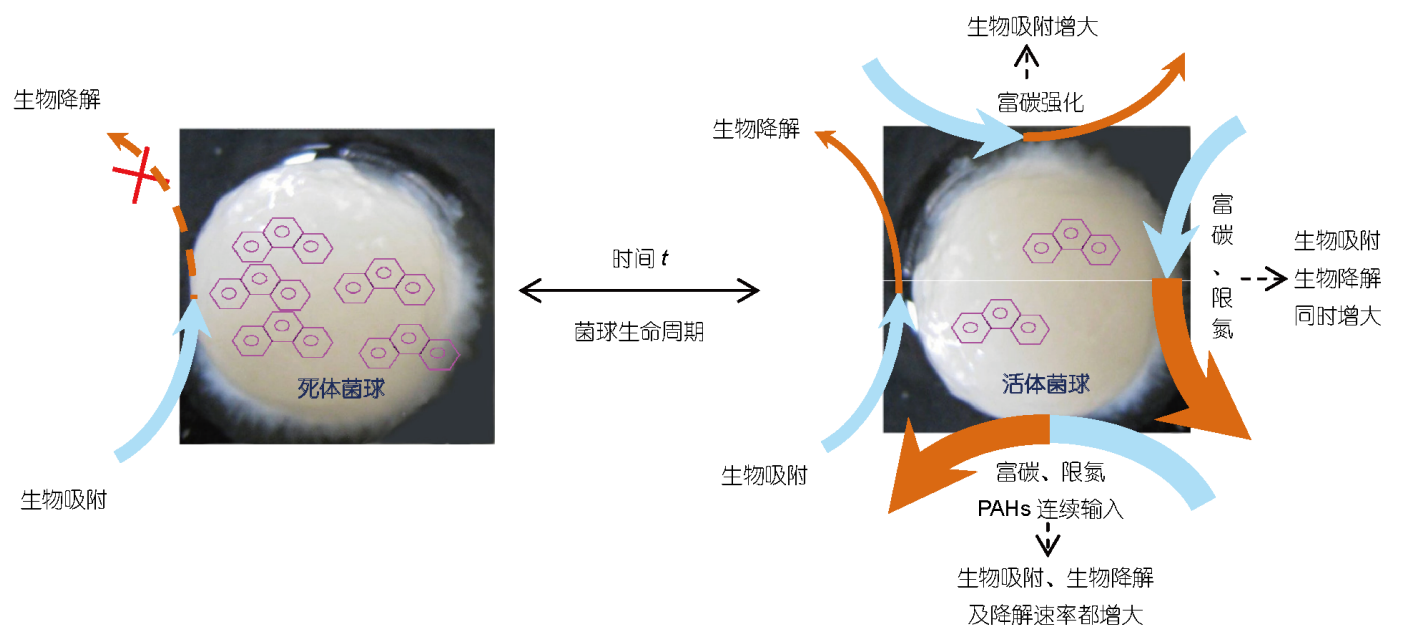

图 4 Pc 死体/活体菌球对 PAHs 的生物吸附、生物降解及其调控作用

降; 同时使得生物降解率减小, 第 5 次时 $\mathrm{C}$ 组的降解 率为 $25.20 \%$, 而 $\mathrm{G}$ 组仅为 $5.12 \%$. 当同时存在碳源强 化时, 这种毒害作用更为明显 ( $\mathrm{H}$ 组), 在第 5 次时, $\mathrm{H}$ 组的降解率仅为 $0.25 \%$. 虽然 $\mathrm{Cu}^{2+}$ 能同时促进 $\mathrm{Pc}$ 对 菲的生物吸附和生物降解, 但连续运行中生物去除 和生物降解则逐渐下降, 因此, $\mathrm{Cu}^{2+}$ 不能作为连续运 行的强化因子.

\section{4 黄狍原毛平革菌生物吸附和生物降解的调控 作用}

$\mathrm{Pc}$ 死体/活体菌球对 PAHs 的生物吸附、生物降 解及其调控作用示意图如图 4 所示. 死体菌球对 PAHs 的去除机制为生物吸附而没有涉及微生物降解, 菌体作为污染物的吸附介质和“储存器”, 因此无法 实现调控. 而活体菌球对污染物同时具有生物吸附 和生物降解作用, 既可以通过强化碳源来增强生物 吸附过程, 降低溶液中 PAHs 浓度; 也可以通过富碳 和限氮途径，同时强化生物吸附和生物降解作用，降 低菌体中积累的 PAHs; 还可以通过富碳和限氮条件 下连续培养、多次输人 PAHs 方式, 在强化生物吸附 和生物降解作用的同时, 提高污染物降解的速度, 实 现 PAHs 的快速去除. 因此, 在污染环境微生物修复
体系和污水活性污泥处理系统中可调控微量持久性 有机污染物的生物吸附和生物降解, 实现 POPs 快速 吸附和有效降解.

\section{3 结论}

(1) Pc 菌体对溶液中菲和萠的去除途径包括生物 吸附和生物降解, 其中生物吸附使 PAHs 在短期内迅 速转移到菌体上, 溶液中的浓度迅速减少; 而生物降 解可以使溶液和菌体中 PAHs 含量随培养时间延长 而降低.

(2) 碳、氮等营养元素可以影响生物吸附和生物 降解过程, 碳是 Pc 生物量的决定因素. 富碳限氮条 件下对 PAHs 的表观去除率和生物降解率最优, $60 \mathrm{~d}$ 数据如下: $99.55 \%$ 和 $92.77 \%$ (菲), $99.47 \%$ 和 $83.97 \%$ (萠).

(3) Pc 死体由于受吸附容量的限制而无法实现连 续运行, 而 Pc 活体菌球在连续运行过程中的去除率 大于死体菌球. 通过富碳和限氮强化, 连续运行 5 次 过程中活体菌球对菲的去除率都保持在 $81 \%$ 以上; 而连续运行 3 次后, 新输人的 $90 \%$ 的菲则通过生物降 解而被去除; 菌体上污染物的残留量降低是连续过 程运行的关键.

\section{参考文献}

1 姚子伟, 江桂斌, 蔡亚歧, 等. 北极地区表层海水中有机污染物和重金属污染的现状. 科学通报, 2002, 47: 1196-1120

2 Breivik K, Alcock R, Li Y F, et al. Primary sources of selected POPs: Regional and global scale emission inventories. Environ Pollut, 2004, 128: 3-16

3 Lohmann R, Breivk K, Dachs J, et al. Global fate of POPs: Current and future research directions. Environ Pollut, 2007, 150: 150-165 
4 Chen B L, Xuan X D, Zhu L Z, et al. Distributions of polycyclic aromatic hydrocarbons in surface waters, sediments and soils of Hangzhou city, China. Water Res, 2004, 38: 3558-3568

5 王小萍, 姚檀栋, 丛志远, 等. 珠穆朗玛峰地区土壤和植被中多环芳烃的含量及海拔梯度分布. 科学通报, 2006, 51: 2517-2525

6 Zhang Y X, Tao S, Shen H Z, et al. Inhalation exposure to ambient polycyclic aromatic hydrocarbons and lung cancer risk of Chinese population. Proc Natl Acad Sci USA, 2009, 106: 21063-21067

7 茹加, 刘会娟, 曲久辉, 等. 新型三油酸甘油酯-活性炭复合吸附剂的性能表征及吸附行为. 科学通报, 2005, 50: 2562-2564

8 陈宝梁, 周丹丹, 朱利中, 等. 生物碳质吸附剂对水中有机污染物的吸附作用及机理. 中国科学 B 辑: 化学, 2008, 38: 530-537

9 Nizzetto L, Macleod M, Borga K, et al. Past, present, and future controls on levels of persistent organic pollutants in the global environment. Environ Sci Technol, 2010, 44: 6526-6531

10 Chen B L, Yuan M X. Enhanced dissipation of polycyclic aromatic hydrocarbons in the presence of fresh plant residues and their extracts. Environ Pollut, 2012, 161: 199-205

11 Semple K T, Doick K J, Jones K C, et al. Defining bioavailability and bioaccessibility of contaminated soil and sediment is complicated. Environ Sci Technol, 2004, 38: 228-231

12 Bamforth S M, Singleton L. Bioremediation of polycyclic aromatic hydrocarbons: Current knowledge and future directions. J Chem Technol Biotechnol, 2005, 80: 723-736

13 Haritash A K, Kaushik C P. Biodegradation aspects of polycyclic aromatic hydrocarbons (PAHs): A review. J Hazard Mater, 2009, 169: 1-15

14 Seo J S, Keum Y S, Li Q X. Bacterial degradation of aromatic compounds. Int J Environ Res Public Health, 2009, 6: 278-309

15 Chen B L, Wang Y S, Hu D H. Biosorption and biodegradation of polycyclic aromatic hydrocarbons in aqueous solutions by a consortium of white-rot fungi. J Hazard Mater, 2010, 179: 845-851

16 Chan S M N, Luan T, Wong M, et al. Removal and biodegradation polycyclic aromatic hydrocarbons by Selenastrum capricornutum Environ Toxicol Chem, 2006, 25: 1772-1779

17 Vijayaraghavan K, Yun Y S. Bacterial biosorbents and biosorption. Biotechnol Adv, 2008, 26: 266-291

18 刘月英, 傅锦冲, 胡洪波, 等. 金霉素链霉菌废菌丝体吸附金 $\left(\mathrm{Au}^{3+}\right)$ 特性的表征. 科学通报, 2001, 46: 1179-1182

19 高大文，文湘华，钱易. 白腐真菌在非灭菌条件下对活性艳红染料的脱色研究. 科学通报, 2004, 49: 1009-1010

20 Aksu Z. Application of biosorption for the removal of organic pollutants: A review. Process Biochem, 2005, 40: 997-1026

21 Park D, Yun Y S, Park J M. The past, present, and future trends of biosorption. Biotechnol Bioprocess Eng, 2010, 15: 86-102

22 丁洁, 王银善, 沈学优, 等. 白腐真菌体对菲和萠的吸附-脱附作用及影响因素. 环境科学学报, 2010, 30: 825-831

23 Stringfellow W T, Alvarez-Cohen L. Evaluating the relationship between the sorption of PAHs to bacterial biomass and biodegradation. Water Res, 1999, 33: 2535-2544

24 Yesilada O, Yildirim S C, Birhanli E, et al. The evaluation of pre-grown mycelia pellets in decolorization of textile dyes during repeated batch process. World J Microbiol Biotechnol, 2010, 26: 33-39

25 Bumpus J A, Tien M, Wright D, et al. Oxidation of persistent environmental-pollutants by a white rot fungus. Science, 1985, 228: $1434-1436$

26 Kim D Y, Rhee Y H. Biodegradation of microbial and synthetic polyesters by fungi. Appl Microbiol Biotechnol, 2003, 61: 300-308

27 Wesenberg D, Kyriakides I, Agathos S N. White-rot fungi and their enzymes for the treatment of industrial dye effluents. Biotechnol Adv, 2003, 22: 161-187

28 Singh D, Chen S. The white-rot fungus Phanerochaete chrysosporium: Conditions for the production of lignin-degrading enzymes. Appl Microbiol Biotechnol, 2008, 81: 399-417

29 Barr D P, Aust S D. Mechanisms white-rot fungi use to degrade pollutants. Environ Sci Technol, 1994, 28: 78-87

30 Loick N, Hobbs P J, Hale M D C, et al. Bioremediation of polyaromatic hydrocarbon (PAH)-contaminated soil by composting. Crit Rev Environ Sci Technol, 2009, 39: 271-332

31 Jeffries T W, Choi S, Krik T K. Nutritional regulation of lignin degradation by Phanerochaete chrysosporium. Appl Environ Microbiol, 1981, 42: 290-296

32 Schhimel J P, Weintraub M N. The implications of exoenzyme activity on microbial carbon and nitrogen limitation in soil: A theoretical model. Soil Biol Biochem, 2003, 35: 549-563

33 Keyser P, Kirk T K, Zeikus J G. Ligninolytic enzyme system of Phanerochaete chrysosporium: Synthesized in the absence of lignin in response to nitrogen starvation. J Bacteriol, 1978, 135: 790-797

34 Binupriya A R, Sathishkumar M, Kavitha D, et al. Experimental and isothermal studies on sorption of Congo Red by modified mycelial biomass of wood-rotting fungus. Clean, 2007, 35: 143-150

35 Dhawale S W, Dhawale S S, Deanross D. Degradation of phenanthrene by Phanerochaete-chrysosporium occurs under ligninolytic as well as nonligninolytic conditions. Appl Microbiol Biotechnol, 1992, 58: 3000-3006 